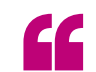

\section{poliovirus} can bind to bacterial LPS, which increases viral stability and infectivity

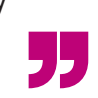

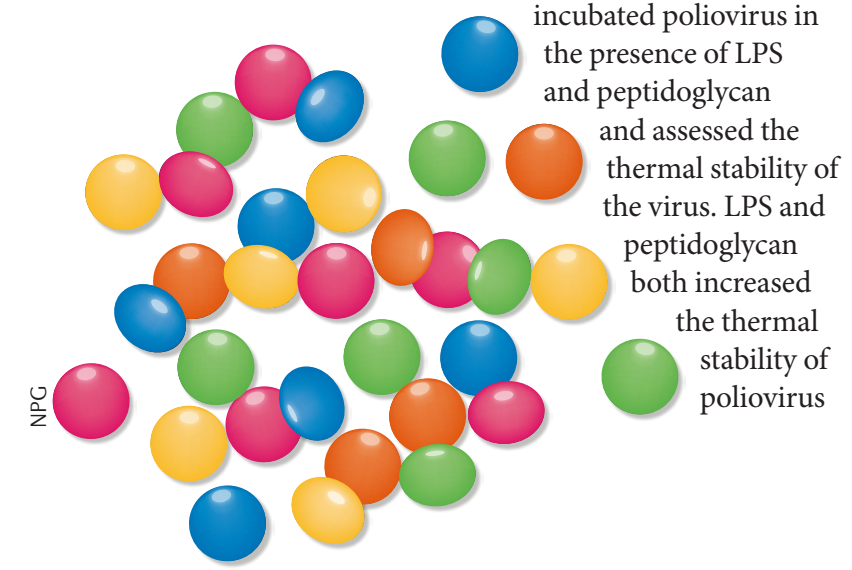
incubated poliovirus in the presence of LPS and peptidoglycan and assessed the thermal stability of the virus. LPS and $h$ increased the thermal stability of poliovirus

$\Rightarrow$ VIRAL INFECTION

\title{
Sugar-coated poliovirus
}

Poliovirus is spread via the faecaloral route and encounters a multitude of microorganisms in the gastrointestinal tract before it establishes infection and disseminates to the nervous system. Previous studies showed that the intestinal microbiota increases the infectivity of poliovirus; however, the mechanism involved was unclear. Robinson et al. now find that the poliovirus capsid protein binds to bacterial lipopolysaccharide (LPS) and that this interaction increases virus stability and attachment to host cells.

Infected individuals shed poliovirus in faeces, and, in the environment, virions often remain in close contact with members of the microbiota or their components, such as LPS. To test whether this interaction influences virion stability, the authors peptidoglycan virions; this was indicated by higher infectivity in cell culture and delayed RNA release compared with poliovirus that had either been incubated in medium or with the oligosaccharide chitohexaose. These results suggest that long bacterial surface components increase poliovirus stability by limiting premature release of the viral genome. Furthermore, the authors showed that LPS and peptidoglycan increased attachment of radiolabelled poliovirus to cells and to purified poliovirus receptor, thus increasing both the stability and the infectivity of poliovirus.

The vaccine strain Sabin serotype 1 is an attenuated poliovirus that has several capsid mutations. The authors determined by site-directed mutagenesis that one of these mutations, T99K, reduces the ability of poliovirus to bind to LPS and to resist degradation in the presence of LPS at $37^{\circ} \mathrm{C}$. However, T99K did not inhibit LPS-mediated stabilization at higher temperatures and it also had no effect on cell attachment, which indicates that this residue is only needed for some aspects of capsid-LPS interactions. Nevertheless, T99K has the potential to affect the environmental stability and the spread of poliovirus via the faecal-oral route. When the authors infected mice with an equal mixture of wild-type and T99K-mutant viruses, the mice shed both viruses in equal amounts in their faeces. However, after incubation of the faeces at room temperature for 96 hours, T99K-mutant virus content was 2.9-fold lower than wild-type virus content, and this value further decreased after peroral infection of secondary mice with virus that had been recovered from the incubated faeces.

Taken together, these results show that poliovirus can bind to bacterial LPS, which increases viral stability and infectivity. The T99K capsid mutation reduces the stabilizing effect of LPS; indeed, in many sewage isolates of Sabin serotype 1, residue 99 has reverted to tyrosine or mutated to another amino acid, which suggests that the T99K mutation - perhaps by reducing LPSbinding - has an environmental fitness cost.

Ursula Hofer

ORIGINAL RESEARCH PAPER Robinson, C. M., Jesudhasan P. R. \& Pfeiffer, J. K. Bacterial lipopolysaccharide binding enhances virion stability and promotes environmental fitness of an enteric virus. Cell Host Microbe 15, 36-46 (2014) 